\title{
A proteomic approach to identify novel disease biomarkers in LCAT deficiency
}

Sara Simonelli ${ }^{\mathrm{a}}$, Alice Ossoli ${ }^{\mathrm{a}}$, Cristina Banfi ${ }^{\mathrm{c}}$, Chiara Pavanello ${ }^{\mathrm{a}}$, Laura Calabresi ${ }^{\mathrm{a}}$, Elisabetta Gianazza ${ }^{\mathrm{b}}$

${ }^{a}$ Centro E. Grossi Paoletti, Dipartimento di Scienze Farmacologiche e Biomolecolari, Università degli Studi di Milano, Italy; ${ }^{b}$ Dipartimento di Scienze Farmacologiche e Biomolecolari, Università degli Studi di Milano, Italy; ${ }^{\mathrm{c} C e n t r o}$ Cardiologico Monzino I.R.C.C.S., Milano, Italy.

\section{Corresponding author:}

Elisabetta Gianazza

Dipartimento di Scienze Farmacologiche e Biomolecolari

Università degli Studi di Milano

via G. Balzaretti 9

I - 20133 Milano - Italia

phone $+39-02-503-18362 /-18256$

email elisabetta.gianazza@unimi.it

\section{Abbreviations}

apoA-I, apolipoprotein A-I;

CE, cholesteryl esters;

ELISA, enzyme-linked immunosorbent assay;

FED, fish-eye disease;

FLD, classical familial LCAT deficiency;

GGE, gel gradient electrophoresis;

HDL, high-density lipoproteins;

HDL-C, cholesterol in high-density lipoproteins;

LCAT, lecithin:cholesterol acyltransferase

LDL-C, cholesterol in low-density lipoproteins 


\section{ABSTRACT}

Genetic LCAT deficiency is a rare recessive autosomal disease due to loss-of-function mutations in the gene coding for the enzyme lecithin:cholesterol acyltransferase (LCAT). Homozygous carriers are characterized by corneal opacity, haemolytic anaemia and renal disease, which represent the first cause of morbidity and mortality in these subjects. Diagnostic and prognostic markers capable of early detecting declining kidney function in these subjects are not available, and the specific serum or urine proteomic signature of LCAT deficient carriers has never been assessed. Taking advantage of a proteomic approach, we performed 2-DE analysis of carriers' plasma and identified proteins present at different concentration in samples from homozygous carriers. Our data confirm the well-known alterations in the concentration of circulating apolipoproteins, with a statistically significant decrease of both apoA-I and apoA-II and a statistically significant increase of apoC-III. Furthermore, we observed increased level of alpha-1antitrypsin, zinc-alpha-2-glycoprotein and retinol-binding protein 4 , and reduced level of clusterin and haptoglobin. Interestingly, only beta but not alpha subunit of haptoglobin is significant reduced in homozygous subjects.

Despite the limited sample size, our findings set the basis for assessing the identified protein in a larger population and for correlating their levels with clinical markers of renal function and anaemia.

\section{Keywords}

LCAT deficiency; apolipoproteins; haptoglobin; haemolysis

\section{Significance}

This investigation defines the effects of LCAT deficiency on the level of the major plasma proteins in homozygous and heterozygous carriers. Increase for some proteins, with different function, together with a drop for haptoglobin, and specifically for haptoglobin beta chains, are reported for the first time as part of a coherent signature.

We are glad to have the opportunity to report our findings on this subject, which is one of the main interests for our research group, when Journal of Proteomics celebrates its $10^{\text {th }}$ anniversary. With its various sections devoted to different areas of research, this journal is a privileged forum for publishing proteomic investigations without restrictions either in sample type or in technical approach. It is as well a privileged forum for reviewing literature data on various topics related to proteomics investigation, as colleagues in our research group have done over the years; by the way, a good share of the reviewed papers were as well reports published in Journal of Proteomics itself. The journal also offers opportunities for focused surveys through thematic issues devoted to a variety of subjects, timely selected for their current relevance in research; it was an honour for colleagues in our group to recently act as editors of one of those. Out of this diverse experience, we express our appreciation for the endeavour of 
Journal of Proteomics in its first 10 years of life - and wish identical and possibly greater success for the time to come.

\section{Highlights}

- The plasma proteome of homozygous and heterozygous carriers of LCAT mutations were analysed.

- Decreased levels of apolipoproteins $v s$ control subjects could be confirmed.

- The next most relevant change observed was a substantial drop in haptoglobin beta chains.

- This finding correlates with altered membrane composition and stability, haemolysis and anaemia.

- A few proteins, with diverse biological function, were found at increased levels in heterozygotes. 


\section{Introduction}

Human lecithin:cholesterol acyltransferase (LCAT) is a plasma glycoprotein responsible of the synthesis of most of the plasma cholesteryl esters (CE) in humans. LCAT plays a central role in the intravascular metabolism of high-density lipoprotein (HDL) and in the determination of the levels of cholesterol associated with them (HDL-C) [1]. Mutations in the LCAT gene cause two LCAT deficiency syndromes, classical familial LCAT deficiency (FLD, MIM\# 245900) and fish-eye disease (FED, MIM\# 136120) [2]. The differential diagnosis of FLD and FED is based on stringent biochemical criteria and is restricted to homozygotes or compound heterozygotes for these mutations. FLD cases have a completely defective cholesterol esterification; as a result, there is very little CE in plasma, and unesterified cholesterol accumulates in all plasma lipoprotein fractions. In FED cases, LCAT does not esterify cholesterol in HDL, which represent the preferred substrate of the enzyme, but does it so in VLDL and LDL: as a result, $\mathrm{CE}$ are present in plasma if at much reduced levels [2].

Genetic LCAT deficiency is characterized by an abnormal plasma lipoprotein profile featuring a low HDL-C level, with mainly small discoidal pre-beta particles, together with the presence of LpX, an abnormal lipoprotein usually absent in plasma and detectable only in some pathological cases [3, 4]. Carriers also present with corneal opacity due to cholesterol accumulation, mild chronic normochromic anaemia, and renal disease. Renal disease, which ultimately progresses to end-stage renal disease, is the major cause of morbidity and mortality in LCAT-deficient carriers [2]. The dramatic alterations in lipoprotein profile, especially the presence of LpX, have been shown to be directly involved in the glomerulosclerosis development [5] but the cause of kidney failure in these subjects is poorly understood.

No specific treatment for renal disease in LCAT deficiency is currently available, and carriers are usually treated symptomatically. The therapy of LCAT deficiency nephropathy mainly aims at delaying the evolution of chronic nephropathy on the basis of available therapies, such as changes in life style and diet and control of complications such as hypertension and proteinuria [2]. FLD patients are often treated by dialysis $[6,7]$; they are candidates for renal transplantation, but the disease can rapidly reoccur in the transplanted tissues within a few years [8].

Not all carriers of LCAT mutations develop renal disease, and the rate of progression of renal disease is unpredictable, even within the same family; some patients rapidly worsen from a mild proteinuria to a rapid deterioration of their renal function [9]. Thus, there is the urgent need for specific diagnostic and prognostic markers, other than traditional measures of renal function, capable of detecting declining kidney function early in time in these individuals in order to address high-risk carriers toward preventive programs and potential therapies.

In this context, proteomic provides useful information for the identification and characterization of alterations in plasma protein levels. Up to now no specific serum or urine proteomic signature of LCAT deficient carriers has been defined. In this work, we applied a proteomic approach to investigate the plasma pattern in homozygous and heterozygous carriers of LCAT deficiency, in comparison with the pattern of non-affected subjects. 


\section{Materials and Methods}

\subsection{Subjects}

The investigation involved a total of 38 carriers of $L C A T$ mutations, all FLD; 13 homozygotes and 25 heterozygotes, and 23 non-affected family members (controls), all belonging to Italian LCAT-deficient families [9]. All of the subjects were fully informed of the modalities and end points of the study and signed an informed consent. Proteomic analysis of plasma was performed on samples from ten carriers, and eight age-matched controls. Five individuals were carriers of two mutant LCAT alleles (four homozygotes and one compound heterozygote, defined homozygotes throughout the paper), while five subjects were heterozygotes; all of them were diagnosed as FLD.

Blood samples were collected, after an overnight fast, into tubes containing $\mathrm{Na}_{2}$-EDTA; plasma was prepared by low speed centrifugation at $4^{\circ} \mathrm{C}$ and stored in aliquots at $-80^{\circ} \mathrm{C}$ until assayed.

\subsection{Biochemical analyses}

Plasma total and unesterified cholesterol, triglyceride and HDL-C levels were determined with standard enzymatic techniques; LDL-C was calculated with the Friedewald's equation. Plasma apolipoprotein levels were measured by immunoturbidimetry on a Roche c311 autoanalyzer. The plasma pre $\beta$-HDL content, expressed as percentage of total plasma apolipoprotein A-I (apoA-I), was determined by native 2-DE, in which agarose gel electrophoresis was followed by nondenaturing gel gradient electrophoresis (GGE), and subsequent immunoblotting for apoA-I [10]. Plasma LCAT concentration was measured by an immunoenzymatic assay [11], and the esterification of cholesterol incorporated into an exogenous standardized substrate (LCAT activity) was measured as previously described [9]. Plasma haptoglobin levels were determined via enzyme-linked immunosorbent assay (ELISA) using the Human Haptoglobin Quantikine ELISA kit (catalogue \#DHAPG0; R\&D Systems, Inc., Minneapolis, USA) according to the manufacturer's instructions.

\subsection{Electrophoretic procedures}

Two-dimensional electrophoresis (2-DE) maps were obtained using the immobilised $\mathrm{pH}$ gradient (IPG)-Dalt method, as previously described $[12,13]$. Plasma $(25 \mu \mathrm{L})$ was diluted with an equal volume of water and reduced with 2\% 2-mercaptoethanol; each plasma sample was loaded at the cathodic end of one laboratory-made focusing strip. The proteins were first resolved according to charge on a non-linear $\mathrm{pH}$ 4-10 IPG [14] in the presence of $8 \mathrm{M}$ urea and $0.5 \%$ carrier ampholytes with an anode-to-cathode distance of $8 \mathrm{~cm}$. The focused proteins were then fractionated according to size by SDS-PAGE on 7.5$17.5 \%$ polyacrylamide gradients on $160 \times 140 \mathrm{~mm}^{2}$ SDS slabs using the discontinuous buffer system of Laemmli [15]. Finally, the proteins were stained with $0.3 \%$ (wt/vol) Coomassie.

The scanned gel patterns were analysed with Image Master Software (Amersham Biosciences, ver. 5.0) and the spectrometry identification of proteins, whose spot volume (i.e., absorbance integrated over area) 
was statistically different among the three groups of subjects, was made as previously described [16].

For haptoglobin phenotyping, equal volumes of human plasma were diluted in sample loading buffer $(1: 100)$ and analysed as previously described [17].

Briefly, levels of haptoglobin subunits were evaluated in plasma diluted in sample loading buffer (1:100) (20 g/l sodium dodecyl sulfate, $100 \mathrm{ml} / 1$ glycerol, $25 \mathrm{mmol} / 1 \mathrm{Tris}$ [pH 6.8], $0.05 \mathrm{~g} / \mathrm{l}$ bromophenol blue, $50 \mathrm{mmol} / 1$ dithiothreitol), and loaded on a $18 \%$ T polyacrylamide gel with Hp standards 1-1 and 2-2 (Sigma-Aldrich, Milan, Italy).

Immunoblotting was performed with a 1:10,000 dilution of polyclonal rabbit anti-human Hp (SigmaAldrich) and a goat anti-rabbit immunoglobulin-G horseradish peroxidase conjugated (Bio-Rad Laboratories, Milan, Italy), diluted 1:10,000 in Tris-buffered saline and Tween 20 (TBST) as secondary antibody. The bands of alpha and beta subunits ( $\sim 20 \mathrm{kDa}$ and $\sim 50 \mathrm{kDa}$, respectively) were detected by ECL and quantified by densitometry using an image analysis software (QuantityOne version 4.5.2; BioRad, Milan, Italy). For each subject, data are reported as the ratio of band volume, after local background subtraction, $v s$ the volume of a normalising sample loaded in each gel, and are expressed in arbitrary units (AU). Inter-assay coefficient of variation was $12.1 \pm 2.9 \%$.

\subsection{Statistical analyses}

Results are presented as mean $\pm \mathrm{SD}$. The trends in plasma protein levels across genotypes were assessed by ANOVA; P values $<0.05$ were considered as statistically significant. Statistical analysis was performed using SPSS version 24.0 software (SPSS Inc., Chicago, USA).

\section{Results}

\subsection{Characteristics of the subjects}

Individuals from the previously reported Italian families with mutations in $L C A T$ gene participated in the study [9]. Only few subjects were submitted to a detailed two-dimensional (2-DE) proteomic investigation: in particular we analysed 5 FLD homozygotes, 5 FLD heterozygotes and 8 non-affected family members who acted as controls. Carriers of $L C A T$ gene mutations, as previously reported, have significantly reduced HDL-C, apoA-I, apoA-II levels, and LCAT mass, with a gene-dose dependent effect. LCAT activity, as expected, was null in homozygous subjects and significantly reduced in heterozygotes while triglyceride levels, unesterified cholesterol, unesterified/total cholesterol and pre $\beta$ HDL were significantly increased (Table 1).

\subsection{2-DE analyses}

The analysis of the plasma proteome of the three groups of subjects was carried out under reducing conditions with $1^{\text {st }}$ dimension in the 4-10 $\mathrm{pH}$ range; Figure 1 shows the 2DE map of a control sample in the top panel and of a LCAT-deficient patient in the bottom panel. As expected, in most cases, we observed spot rows rather than single spots, each protein species [18] resulting from a specific set of post- 
translational modifications $[19,20]$. When relevant, the statistic analysis on spot volumes was made both on the values of each individual spot and of the whole spot row.

The proteins differing in volume in one or more spots, corresponding to one or more of their PTMspecies or of their proteolytic fragments, in a patients $v s$ controls comparison, are outlined in the top panel; their numbering corresponds to that of the entries in Table 2 and in Supplementary Table 1, which contain the identifications made possible by MS analysis of the picked spots. Table 2 summarizes as well the significant changes in spot abundance pointed out by the statistical treatment of the 2-DE densitometric data.

From the above comparison, significantly lower levels of apolipoprotein A-I and apolipoprotein A-II were highlighted, as expected, in homozygous subjects with respect to controls. In addition, in homozygotes, we found increased plasma levels of several proteins such as alpha-1-antitrypsin, zincalpha-2-glycoprotein, retinol-binding protein 4 and transthyretin, and significantly lower levels of the beta chain of haptoglobin.

\subsection{Haptoglobin}

Plasma haptoglobin levels were measured in a larger group of subjects that included 13 FLD homozygotes, 25 FLD heterozygotes and 23 controls (Supplementary Table 2). Plasma protein concentration both in controls and in patients was within the reported reference range (32-205 mg/dL) and the ELISA measurement did not confirm the reduction of haptoglobin concentration in homozygous subjects, observed in 2-DE analysis. Indeed, carriers of $L C A T$ gene mutations showed plasma haptoglobin levels comparable to controls (controls $74.0 \pm 27.3 \mathrm{mg} / \mathrm{dL}$; heterozygotes, $80.8 \pm 35.7 \mathrm{mg} / \mathrm{dL}$; homozygotes, $75.7 \pm 65.2 \mathrm{mg} / \mathrm{dL}$; $P$ for trend $=0.58$ ).

In order to find a rationale for the above discrepancy, we investigated by immunoblotting after 1-DE the abundance of the haptoglobin subunits (alpha and beta), characterized by different molecular weights. With this approach, we observed that homozygous subjects are characterized by a significant reduction of the beta subunit (homozygotes, $0.99 \pm 0.38 \mathrm{AU}$; heterozygotes, $1.43 \pm 0.14 \mathrm{AU}$; controls $1.44 \pm 0.22 \mathrm{AU}$; $P$ for trend $=0.016$; Figure 2) but not of the alpha subunit.

\section{Discussion}

The proteomic investigation we describe in this report affords for the first time a comprehensive evaluation of all major plasma components in homozygous carriers of LCAT mutations that result in the virtual absence from the circulation of the enzyme in its active form [1].

Our data on homozygous carriers fully confirm the well-known alterations in the concentration of circulating apolipoproteins, with a statistically significant decrease of both apoA-I and apoA-II and a statistically significant increase of apoC-III [21]. The main interest, however, of our approach is the indication it provides for altered concentrations of serum components other than apolipoproteins and 
related to clinical manifestations of the disease, which include anaemia and renal disease. Specifically haptoglobin and clusterin decrease, while alpha-1-antitrypsin, zinc-alpha-2-glycoprotein and retinolbinding protein 4 increase in homozygous carriers.

Haptoglobin concentration is influenced by diet, and specifically by amount and composition of dietary fat: it was found significantly up-regulated in animals receiving a high-fat, high-cholesterol chow [22] whereas it was significantly down-regulated in human volunteers receiving omega-3 supplementation [23]. In more detail, haptoglobin is characterized by a molecular heterogeneity, which includes three major phenotypes: Hpt 1-1, Hpt 2-1 and Hpt 2-2. The various phenotypes perform differently in connection with lipid metabolism: when tested in in vitro and in vivo models of diabetes mellitus, reverse cholesterol transport appears much more reduced in Hp2-2 than in Hp1-1 mice [24]. The rationale for testing diabetic animals in the latter trial is that a differential prevalence of coronary heart disease - one of the sequels of altered cholesterol transport - is observed in human patients depending on haptoglobin phenotype, with opposite trend in diabetic (lower in 2-1) and in non-diabetic subjects (higher in 2-1) [25].

In our 2-DE experiments, a statistically significant decrease in homozygous $v s$ control subjects is observed for as many as 6 spots identified as haptoglobin or better, from their position in the $\mathrm{pI} / \mathrm{M}_{\mathrm{r}}$ map, for haptoglobin beta chains. The finding was confirmed by an independent approach based on molecular recognition, namely immunoblotting after 1-DE separation of the proteins. On the contrary, ELISA does not distinguish the different haptoglobin subunits.

The finding of reduced circulating haptoglobin is related to normochromic haemolytic anaemia detected in FLD subject. In erythrocytes, the increase in free cholesterol and phosphatidylcholine and the decrease in phosphatidylethanolamine affect membrane fluidity; the lysis of fragile cells eventually leads to normochromic normocytic anaemia; hemoglobin is released meanwhile [26-28]. Haptoglobin binds free hemoglobin and the complex is cleared from the circulation [29]. In connection with iron homeostasis, the 2-2 phenotype is associated with lower affinity for hemoglobin and lower antioxidant capacity, which results in higher sideraemia, higher ferritin and lower transferrin concentrations, and higher transferrin saturation than in the 1-1 and 2-1 phenotypes [30].

The formation of the complex between hemoglobin and haptoglobin involves a direct contact between the alfa-beta protomer of the former and the beta chain of the latter. This was first deduced from binding experiments using either synthetic peptides [31,32] or isolated whole subunits [33] and has been demonstrated in crystallographic experiments with both bovine [34] and human [35] proteins. It is not obvious to proceed from this evidence to our findings of a selective decrease of the circulating levels of just the beta subunits of haptoglobin, as we have evaluated both in the densitometric measurements on the spots resolved by electrophoresis and by immunoblotting. In fact, haptoglobin alpha and beta chain, bound to one another by disulfide bridges, result from the proteolytic cleavage of a single polypeptide transcript [36], which should ensure a stoichiometric synthesis. Also the mechanism of the clearance of the complex, requiring the internalization via the monocyte/macrophage scavenger receptor CD163 [37] and the transfer to early endosomes [38] implies the degradation to heme, bioactive peptides, and amino 
acids, without any selectivity for either of the chain components. We could not find literature data about a varying effect on the different subunits of the condition we are considering here, hemolysis. However, Fernandez-Costa et al. report at least one example of uneven regulation of the haptoglobin chains [39]. By immunoblot on osteoarthritis patient's sera, these authors detected an increase of the beta chain by 2.22-fold $v s$ control subjects; instead, the alpha chain increased only by 1.4 -fold when in the 1 phenotype and decreased 0.7 -fold when in the 2 phenotype. While without statistical significance per se the latter result was duplicated by quantitation through the multiple reaction monitoring technology (digestion, nanoHPLC, MS).

Both haptoglobin and clusterin/apoJ, another protein changed in proteomic pattern of homozygous carriers, may be present in the circulation either in soluble form or associated with HDL. Actually, haptoglobin-related protein exists solely as a component of a minor subspecies of large high-density lipoproteins (HDL3) containing apolipoprotein L-I. Its assembly into the lipoprotein particle is mediated by the retained N-terminal signal peptide, an unusual feature for a secreted protein and the major difference between haptoglobin-related protein and haptoglobin. The 18-amino acid signal peptide interacts directly with the hydrocarbon region of lipids: lipid fluidity hastens the interaction while lipid rigidity stabilizes the association [40]. Haptoglobin is able as well to bind to lipoproteins through its interaction with apoA-I [41] and apoE [42].

Two of the three significantly increased protein in homozygous carriers, i.e. retinol-binding protein 4 and zinc-alpha-2-glycoprotein have been described altered in chronic kidney diseases.

Circulating retinol-binding protein 4 appears positively correlated with serum creatinine levels and inversely correlated with glomerular filtration rates [43-46]; no correlation is observed instead with another sign of glomerular dysfunction, microalbuminuria [43], or with a different type of long-term sequel of diabetes, atherosclerosis, as assessed by carotid intima-media thickness [45]. Plasma levels of retinol-binding protein 4 decrease after kidney transplantation to patients with end-stage renal disease [46]. In carriers of genetic LCAT deficiency retinol-binding protein 4 increases in plasma with a genedose dependent effect.

In patients with chronic kidney disease, plasma concentration of zinc-alpha-2-glycoprotein increases with the progression of the disease and the decline of glomerular filtration rate [47]. The observation that mice (-/-) for the corresponding gene develop significantly more kidney fibrosis in models of kidney and heart injury suggests that this protein exerts an antifibrotic effect that is duplicated in vitro by sera of patients with high levels of zinc-alpha-2-glycoprotein [48]. Zinc-alpha-2-glycoprotein is significantly increased in plasma of homozygous and compound heterozygous carriers of genetic LCAT deficiency.

\section{Conclusions}


The concentration of any given protein at any given time in any given compartment results from the compound effects not only of synthesis and catabolism but also of distribution from/to any other compartment. The latter may be ruled solely by the physic-chemical properties of the protein or be mediated by its interaction with other proteins, to form assemblies in solution and/or receptor-ligand complexes at the cell surface. Examples of counterintuitive explanations for analytical findings are provided in routine clinical biochemistry tests by increase in circulating myoglobin due to decreased glomerular filtration and by decrease in $\mathrm{C} 3$ and/or $\mathrm{C} 4$ due to increased consumption after activation of the complement pathway(s). Changes in protein concentration independent from regulation of protein synthesis are observed also in the homozygous/doubly heterozygous carriers of LCAT mutations.

A low-grade inflammatory condition is an expected outcome in the presence of reduced levels of apoA-I (some human data: [49, 50]; some animal data: [51, 52]). The observation of increased concentration for alpha-1-antitrypsin in the homozygous/doubly heterozygous carriers of LCAT mutations confirms the expectation for its increased synthesis in these subjects. Up-regulation is to be assumed as well for haptoglobin; the finding, instead, of a lower concentration in the carriers must be caused by the overlap with a process consuming haptoglobin. Such an interfering phenomenon is easily identified in the scavenging of hemoglobin as it leaks from erythrocytes; in turn, haemolysis is the known outcome of altered membrane lipid composition in the absence of LCAT activity.

Despite the limited sample size, our finding poses the base to investigate the identified protein in a larger population and correlate them with clinical markers of the disease (renal function and anemia).

\section{Conflict of interest}

None

\section{Acknowledgements}

The authors thank Erica Gianazza for her technical support.

This work was supported in part by Telethon Italy (GGP14125 to L.C.) and by grants from MIUR Progetto Eccellenza. 


\begin{tabular}{|c|c|c|c|c|}
\hline & \multicolumn{3}{|c|}{ Carriers of LCAT deficiency } & \multirow[t]{2}{*}{$P$ trend } \\
\hline & Controls & Heterozygote & Homozygotes & \\
\hline$N$ & 8 & 5 & 5 & \\
\hline $\operatorname{Sex}(m / f)$ & $4 / 4$ & $3 / 2$ & $4 / 1$ & \\
\hline Age (years) & $36.4 \pm 11.1$ & $34.6 \pm 12.5$ & $34.2 \pm 3.1$ & 0.917 \\
\hline Total Cholesterol (mg/dL) & $180.1 \pm 22.1$ & $148.8 \pm 34.1$ & $195.4 \pm 106.1$ & 0.264 \\
\hline Unesterified Cholesterol (mg/dL) & $55.9 \pm 7.3$ & $50.0 \pm 11.1$ & $181.4 \pm 92.4$ & 0.006 \\
\hline Unesterified/total Cholesterol & $0.29 \pm 0.02$ & $0.34 \pm 0.03$ & $0.94 \pm 0.06$ & $<0.001$ \\
\hline LDL-Cholesterol (mg/dL) & $100.7 \pm 26.3$ & $78.3 \pm 26.7$ & $132.3 \pm 78.7$ & 0.348 \\
\hline HDL-Cholesterol (mg/dL) & $63.9 \pm 15.6$ & $43.8 \pm 19.6$ & $9.0 \pm 4.8$ & $<0.001$ \\
\hline Triglycerides (mg/dL) & $77.9 \pm 45.1$ & $142.0 \pm 95.4$ & $254.6 \pm 150.4$ & 0.05 \\
\hline Apolipoprotein A-I (mg/dL) & $125.4 \pm 27.2$ & $94.6 \pm 22.1$ & $39.8 \pm 8.8$ & 0.003 \\
\hline Apolipoprotein A-II (mg/dL) & $31.6 \pm 6.4$ & $28.0 \pm 3.8$ & $7.8 \pm 4.4$ & $<0.001$ \\
\hline Apolipoprotein B (mg/dL) & $83.1 \pm 18.2$ & $82 \pm 24.0$ & $54.8 \pm 25.0$ & 0.113 \\
\hline preß-HDL (\% of apoA-I) & $12.8 \pm 1.7$ & $19.5 \pm 3.1$ & $44.8 \pm 9.3$ & 0.002 \\
\hline LCAT mass $(\mu \mathrm{g} / \mathrm{mL})$ & $5.2 \pm 1.7$ & $3.85 \pm 1.26$ & $1.55 \pm 0.68$ & 0.002 \\
\hline LCAT activity (nmol/mL/h) & $40.3 \pm 7.4$ & $16.8 \pm 10.3$ & nd & $<0.001$ \\
\hline
\end{tabular}

Table 1

Demographic and lipid/lipoprotein profile of carriers of LCAT deficiency

Data are reported as mean \pm SD. $n d=$ not detectable 
Table 2

List of plasma proteins identified by MS differently expressed among groups of subjects

\begin{tabular}{|c|c|c|c|c|c|}
\hline Spot number & Protein & $\begin{array}{c}\text { Uniprot } \\
\text { accession \# }\end{array}$ & Controls & Heterozygotes & Homozygotes \\
\hline 1 & Alpha-1-antitrypsin & P01009 & $1.20 \pm 0.79$ & $1.28 \pm 0.54$ & $2.19 \pm 0.92^{*}$ \\
\hline 2 & Zinc-alpha-2-glycoprotein & P25311 & $0.51 \pm 0.23$ & $0.48 \pm 0.18$ & $1.05 \pm 0.55^{*}, \S$ \\
\hline 3 & Haptoglobin & P00738 & $0.31 \pm 0.16$ & $0.22 \pm 0.06$ & $0.00 \pm 0.00^{*}$ \\
\hline 4 & Haptoglobin & P00738 & $0.84 \pm 0.34$ & $0.58 \pm 0.24$ & $0.00 \pm 0.00^{*}$ \\
\hline $5+6+7+8$ & $\begin{array}{l}\text { Haptoglobin }+ \text { Serum } \\
\text { albumin }\end{array}$ & $\mathrm{P} 00738+\mathrm{P} 02768$ & $5.37 \pm 1.34$ & $5.35 \pm 0.77$ & $3.18 \pm 1.64^{*}, \S$ \\
\hline 9 & Complement $\mathrm{C} 3$ & P01024 & $0.04 \pm 0.08$ & $0.36 \pm 0.20^{\#}$ & $0.00 \pm 0.00^{\S}$ \\
\hline 10 & Zinc-alpha-2-glycoprotein & P25311 & $1.12 \pm 0.31$ & $1.14 \pm 0.30$ & $1.64 \pm 0.72^{*}, \S$ \\
\hline 11 & $\begin{array}{c}\text { Zinc-alpha-2-glycoprotein } \\
\text { or Haptoglobin }\end{array}$ & $\begin{array}{c}\text { P25311 } \\
\text { or P00738 }\end{array}$ & $0.30 \pm 0.10$ & $0.32 \pm 0.14$ & $1.10 \pm 1.14^{*}, \S$ \\
\hline 12 & Clusterin & P10909 & $0.11 \pm 0.04$ & $0.13 \pm 0.06$ & $0.00 \pm 0.00^{*}, \S$ \\
\hline 13 & Apolipoprotein A-I & P02647 & $0.19 \pm 0.08$ & $0.24 \pm 0.17$ & $0.00 \pm 0.00^{\S}$ \\
\hline 14 & Apolipoprotein A-I & P02647 & $2.43 \pm 0.83$ & $2.00 \pm 1.24$ & $0.66 \pm 0.68^{*}$ \\
\hline 15 & Apolipoprotein A-I & P02647 & $10.03 \pm 2.88$ & $14.11 \pm 9.39$ & $4.21 \pm 1.74^{*}, \S$ \\
\hline 16 & Apolipoprotein A-I & P02647 & $0.67 \pm 0.39$ & $0.32 \pm 0.19$ & $1.17 \pm 0.38^{\S}$ \\
\hline $17+18$ & Retinol-binding protein 4 & $\mathrm{P} 02753$ & $0.94 \pm 0.59$ & $1.17 \pm 0.92$ & $2.17 \pm 1.67^{*}$ \\
\hline 19 & $\begin{array}{c}\text { Transthyretin }+ \text { Serum } \\
\text { albumin }\end{array}$ & $\mathrm{P} 02766+\mathrm{P} 02768$ & $0.02 \pm 0.04$ & $0.15 \pm 0.10^{\#}$ & $0.00 \pm 0.00$ \\
\hline 20 & Transthyretin & P02766 & $0.38 \pm 0.13$ & $0.85 \pm 0.38^{\#}$ & $1.03 \pm 0.40^{*}$ \\
\hline 21 & Apolipoprotein A-II & $\mathrm{P} 02652$ & $5.95 \pm 2.25$ & $5.76 \pm 3.15$ & $0.86 \pm 0.47^{*}, \S$ \\
\hline
\end{tabular}

${ }^{*} \mathrm{P}<0.05$ Homozygotes vs controls; ${ }^{{ }_{\mathrm{P}}}<0.05$ Homozygotes vs heterozygotes; ${ }^{\#} \mathrm{P}<0.05$ Heterozygotes $v s$ controls 


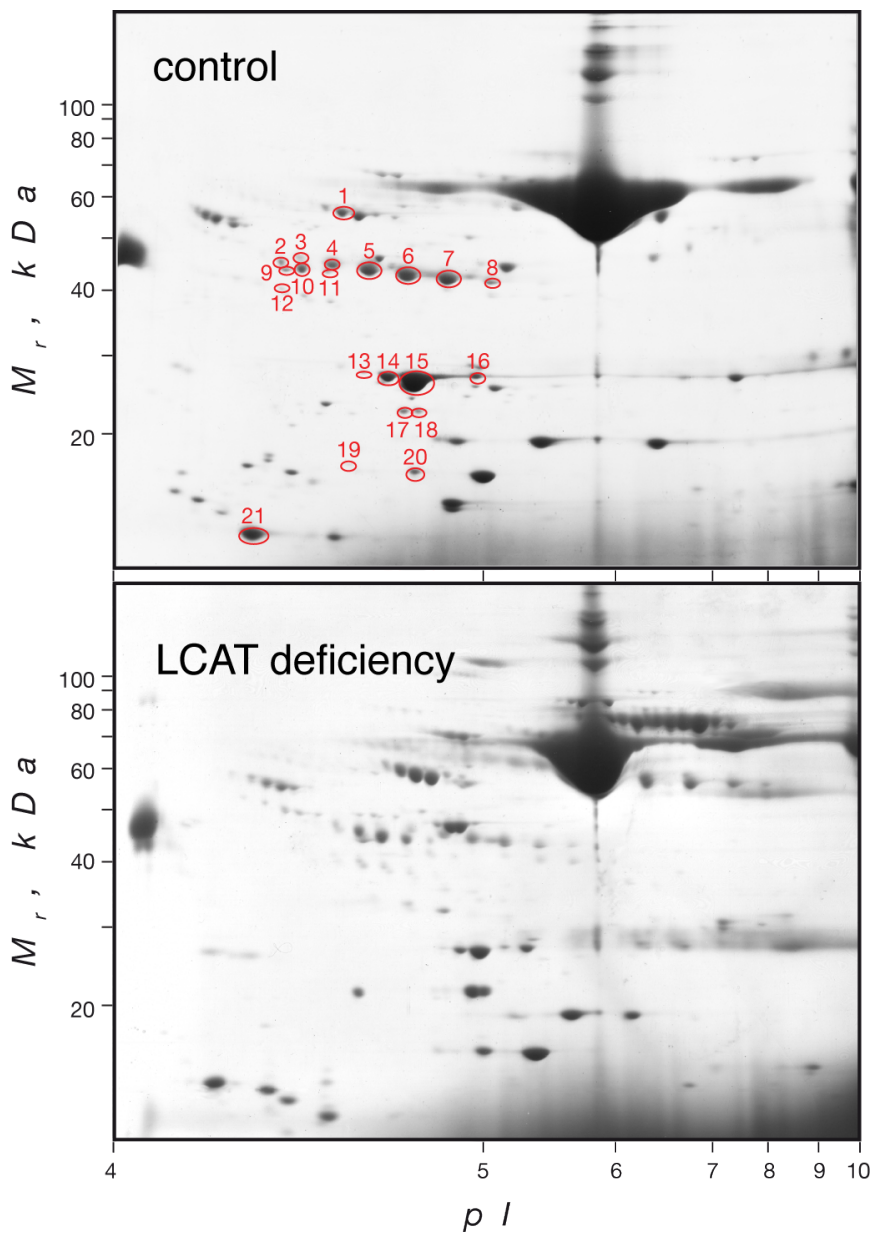

Fig. 1. 2DE map of plasma under reducing conditions: control sample in top panel, LCAT-deficient sample in bottom panel. The first dimension is on a 4-10 non linear IPG, and the second dimension is on polyacrylamide gradient gels (7.5-17.5\%). Spots selected for MS analyses are highlighted, and the entry numbers refer to Table 2 and Supplementary Table 1. 


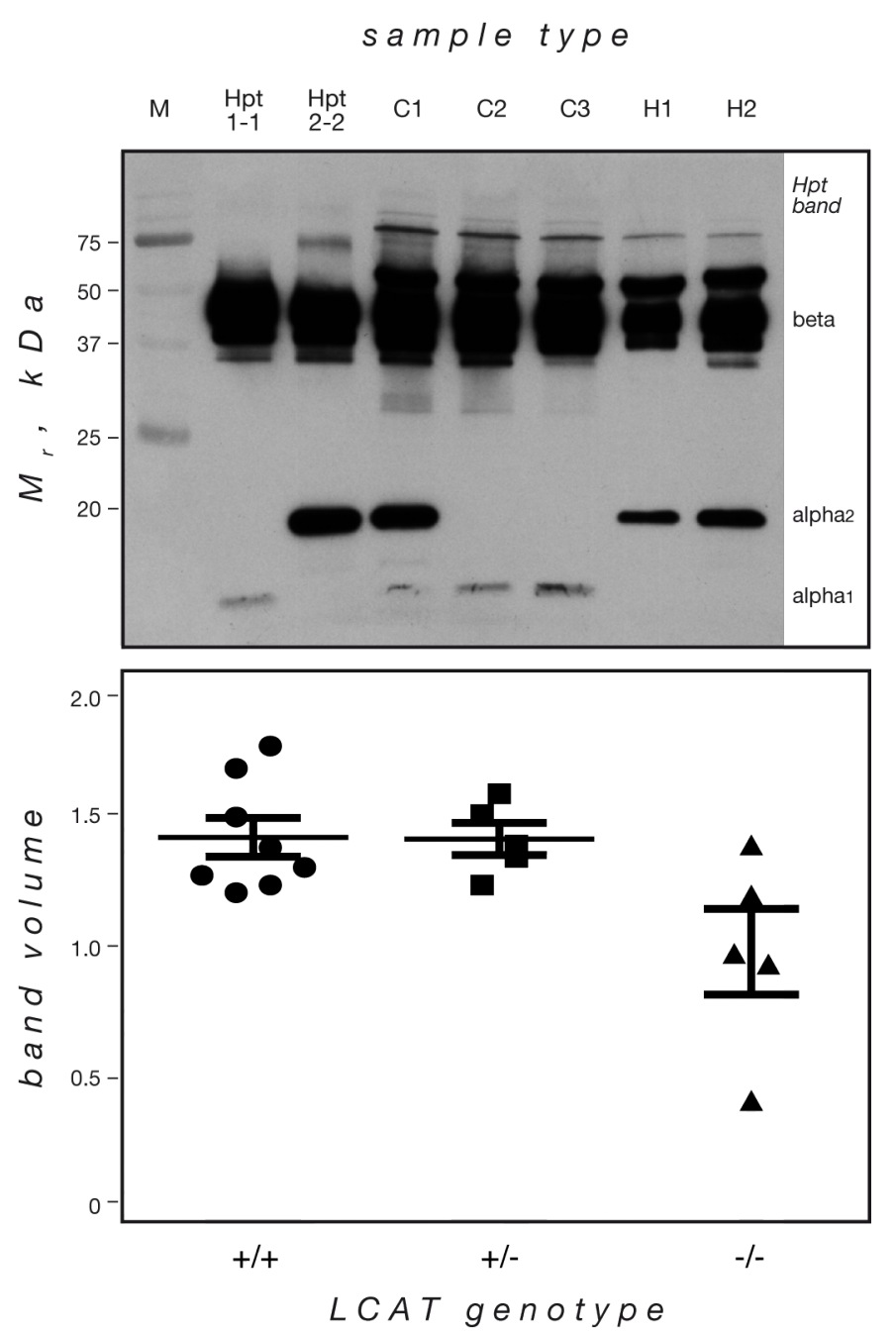

Fig. 2. Plasma $\beta$-subunit levels of haptoglobin were evaluated by immunoblotting, in carriers of LCAT gene mutations $(n=5)$ and in non-affected family members $(n=8)$. A representative immunoblot is shown in the top panel: $\mathrm{M}$ lane contains $\mathrm{M}_{r}$ markers, Hpt 1-1 and 2-2 lanes contain commercial samples with the corresponding phenotypes. The top panel plots the quantitative data from densitometric analysis.

\section{Bibliography}

[1] Calabresi L, Simonelli S, Gomaraschi M, Franceschini G. Genetic lecithin:cholesterol acyltransferase deficiency and cardiovascular disease. Atherosclerosis. 2012;222:299-306.

[2] Santamarina-Fojo S, Hoeg JM, Assmann G, Brewer HBRJ. Lecithin cholesterol acyltransferase deficiency and fish eye disease. In: Scriver CR, Beaudet AL, Sly WS, Valle D, editors. The Metabolic and Molecular Bases of Inherited Diseases. New York: McGraw-Hill; 2001. p. 2817-33.

[3] Ahsan L, Ossoli A, Freeman L, Vaisman B, Amar MJ, Shamburek RD, et al. Role of Lecithin:Cholesterol Acyltransferase in HDL Metabolism and Atherosclerosis. In: Komoda T, editor. The HDL Handbook (Second Edition): Biological Functions and Clinical Implications. Amsterdam: Elsevier Inc.; 2014. p. 159-94.

[4] Ritland S. The abnormal "lipoprotein of cholestasis", lipoprotein-X. Scand J Gastroenterol. 1975;10:785-9.

[5] Ossoli A, Neufeld EB, Thacker SG, Vaisman B, Pryor M, Freeman LA, et al. Lipoprotein X Causes Renal Disease in LCAT Deficiency. PLoS ONE. 2016;11:e0150083.

[6] Tsuchiya Y, Ubara Y, Hiramatsu R, Suwabe T, Hoshino J, Sumida K, et al. A case of familial lecithin-cholesterol acyltransferase deficiency on hemodialysis for over 20 years. Clinical nephrology. 2011;76:492-8. 
[7] Weber CL, Frohlich J, Wang J, Hegele RA, Chan-Yan C. Stability of lipids on peritoneal dialysis in a patient with familial LCAT deficiency. Nephrology, dialysis, transplantation : official publication of the European Dialysis and Transplant Association - European Renal Association. 2007;22:2084-8.

[8] Strom EH, Sund S, Reier-Nilsen M, Dorje C, Leren TP. Lecithin: Cholesterol Acyltransferase (LCAT) Deficiency: renal lesions with early graft recurrence. Ultrastructural pathology. 2011;35:139-45.

[9] Calabresi L, Pisciotta L, Costantin A, Frigerio I, Eberini I, Alessandrini P, et al. The molecular basis of lecithin:cholesterol acyltransferase deficiency syndromes: a comprehensive study of molecular and biochemical findings in 13 unrelated Italian families. Arterioscler Thromb Vasc Biol. 2005;25:1972-8.

[10] Favari E, Lee M, Calabresi L, Franceschini G, Zimetti F, Bernini F, et al. Depletion of pre-beta-high density lipoprotein by human chymase impairs ATP-binding cassette transporter A1- but not scavenger receptor class B type I-mediated lipid efflux to high density lipoprotein. J Biol Chem. 2004;279:9930-6.

[11] Murakami T, Michelagnoli S, Longhi R, Gianfranceschi G, Pazzucconi F, Calabresi L, et al. Triglycerides are major determinants of cholesterol esterification/transfer and HDL remodeling in human plasma. Arter Thromb Vasc Biol. 1995; 15:1819-28.

[12] Gianazza E. Casting immobilized pH gradients. In: Walker JM, editor. The Protein Protocol Handbook. 3rd ed. Totowa: Humana Press; 2009. p. 305-22.

[13] Eberini I, Gianazza E, Breghi L, Klugmann S, Calabresi L, Gomaraschi M, et al. Apolipoprotein A-I breakdown is induced by thrombolysis in coronary patients. Annals of medicine. 2007;39:306-11.

[14] Gianazza E, Giacon P, Sahlin B, Righetti PG. Non-linear pH courses with immobilized pH gradients. Electrophoresis. 1985;6:53-6.

[15] Laemmli UK. Cleavage of structural proteins during the assembly of the head of bacteriophage T4. Nature. 1970;227:680-5 [16] De Benedetti S, Gianazza E, Banfi C, Marocchi A, Lunetta C, Penco S, et al. Serum Proteome in a Sporadic Amyotrophic Lateral Sclerosis Geographical Cluster. Proteomics Clin Appl. 2017;11.

[17] Pontone G, Andreini D, Guaricci AI, Guglielmo M, Baggiano A, Muscogiuri G, et al. Association Between Haptoglobin Phenotype and Microvascular Obstruction in Patients With ST-Segment Elevation Myocardial Infarction: A Cardiac Magnetic Resonance Study. JACC Cardiovascular imaging. 2018.

[18] Jungblut PR, Schluter H. Towards the analysis of protein species: an overview about strategies and methods. Amino Acids. 2011;41:219-22.

[19] Jungblut PR. Back to the future--the value of single protein species investigations. Proteomics. 2013;13:3103-5.

[20] Gianazza E, Parravicini C, Primi R, Miller I, Eberini I. In silico prediction and characterization of protein post-translational modifications. J Proteomics. 2016;134:65-75.

[21] Saeedi R, Li M, Frohlich J. A review on lecithin:cholesterol acyltransferase deficiency. Clin Biochem. 2015;48:472-5.

[22] Gianazza E, Sensi C, Eberini I, Gilardi F, Giudici M, Crestani M. Inflammatory serum proteome pattern in mice fed a highfat diet. Amino Acids. 2013;44:1001-8.

[23] de Roos B, Geelen A, Ross K, Rucklidge G, Reid M, Duncan G, et al. Identification of potential serum biomarkers of inflammation and lipid modulation that are altered by fish oil supplementation in healthy volunteers. Proteomics. 2008;8:1965-74.

[24] Asleh R, Miller-Lotan R, Aviram M, Hayek T, Yulish M, Levy JE, et al. Haptoglobin genotype is a regulator of reverse cholesterol transport in diabetes in vitro and in vivo. Circ Res. 2006;99:1419-25.

[25] Levy AP, Larson MG, Corey D, Lotan R, Vita JA, Benjamin EJ. Haptoglobin phenotype and prevalent coronary heart disease in the Framingham offspring cohort. Atherosclerosis. 2004;172:361-5.

[26] Gillett MP, Teixeira V, Dimenstein R. Decreased plasma lecithin:cholesterol acyltransfer and associated changes in plasma and red cell lipids in uraemia. Nephrology, dialysis, transplantation : official publication of the European Dialysis and Transplant Association - European Renal Association. 1993;8:407-11.

[27] Lambert G, Sakai N, Vaisman BL, Neufeld EB, Marteyn B, Chan CC, et al. Analysis of glomerulosclerosis and atherosclerosis in lecithin cholesterol acyltransferase-deficient mice. J Biol Chem. 2001;276:15090-8.

[28] Suda T, Akamatsu A, Nakaya Y, Masuda Y, Desaki J. Alterations in erythrocyte membrane lipid and its fragility in a patient with familial lecithin:cholesterol acyltrasferase (LCAT) deficiency. The journal of medical investigation : JMI. 2002;49:147-55. [29] Ratanasopa K, Chakane S, Ilyas M, Nantasenamat C, Bulow L. Trapping of human hemoglobin by haptoglobin: molecular mechanisms and clinical applications. Antioxid Redox Signal. 2013;18:2364-74.

[30] Van Vlierberghe H, Langlois M, Delanghe J. Haptoglobin polymorphisms and iron homeostasis in health and in disease. Clin Chim Acta. 2004;345:35-42.

[31] Kazim AL, Atassi MZ.

Haemoglobin binding with haptoglobin. Localization of the haptoglobin-binding site on the $\alpha$-chain of human haemoglobin. Biochem J. 1981;197:507-10.

[32] Yoshioka N, Atassi MZ. Haemoglobin binding with haptoglobin. Localization of the haptoglobin-binding sites on the betachain of human haemoglobin by synthetic overlapping peptides encompassing the entire chain. Biochem J. 1986;234:453-6.

[33] Odegaard B, Atassi MZ. Haptoglobin-hemoglobin binding involves the heavy chain of haptoglobin and the alpha and beta chains of hemoglobin. J Prot Chem. 1984;3:287-92.

[34] Andersen CB, Torvund-Jensen M, Nielsen MJ, de Oliveira CL, Hersleth HP, Andersen NH, et al. Structure of the haptoglobin-haemoglobin complex. Nature. 2012;489:456-9.

[35] Lane-Serff H, MacGregor P, Lowe ED, Carrington M, Higgins MK. Structural basis for ligand and innate immunity factor uptake by the trypanosome haptoglobin-haemoglobin receptor. eLife. 2014;3:e05553.

[36] Yoshioka Y, Atassi MZ. Prohaptoglobin is proteolytically cleaved in the endoplasmic reticulum by the complement C1r-like protein. Proc Natl Acad Sci USA. 2004;101:14390-5,

[37] Schaer CA, Schoedon G, Imhof A, Kurrer MO, Schaer DJ. Constitutive endocytosis of CD163 mediates hemoglobin-heme uptake and determines the noninflammatory and protective transcriptional response of macrophages to hemoglobin. Circ Res. 2006;99:943-50.

[38] Fruitier I, Garreau I, Lacroix A, Cupo A, Piot JM. Proteolytic degradation of hemoglobin by endogenous lysosomal proteases gives rise to bioactive peptides: hemorphins. FEBS Lett. 1999;447:81-6. 
[39] Fernandez-Costa C, Calamia V, Fernandez-Puente P, Mateos J, Rocha B, Lourido L, et al. The three chains of haptoglobin as potential diagnostic markers of osteoarthritis disease. Osteoarthritis and Cartilage. 2014;22:S72.

[40] Harrington JM, Nishanova T, Pena SR, Hess M, Scelsi CL, Widener J, et al. A retained secretory signal peptide mediates high density lipoprotein (HDL) assembly and function of haptoglobin-related protein. J Biol Chem. 2014;289:24811-20.

[41] Spagnuolo MS, Cigliano L, D'Andrea LD, Pedone C, Abrescia P. Assignment of the binding site for haptoglobin on apolipoprotein A-I. J Biol Chem. 2005;280:1193-8.

[42] Cigliano L, Pugliese CR, Spagnuolo MS, Palumbo R, Abrescia P. Haptoglobin binds the antiatherogenic protein apolipoprotein $\mathrm{E}$ - impairment of apolipoprotein $\mathrm{E}$ stimulation of both lecithin:cholesterol acyltransferase activity and cholesterol uptake by hepatocytes. The FEBS journal. 2009;276:6158-71.

[43] Cabre A, Lazaro I, Girona J, Manzanares J, Marimon F, Plana N, et al. Retinol-binding protein 4 as a plasma biomarker of renal dysfunction and cardiovascular disease in type 2 diabetes. Journal of internal medicine. 2007;262:496-503.

[44] Henze A, Frey SK, Raila J, Scholze A, Spranger J, Weickert MO, et al. Alterations of retinol-binding protein 4 species in patients with different stages of chronic kidney disease and their relation to lipid parameters. Biochem Biophys Res Commun. 2010;393:79-83.

[45] Chu CH, Lam HC, Lee JK, Lu CC, Sun CC, Cheng HJ, et al. Elevated serum retinol-binding protein 4 concentrations are associated with chronic kidney disease but not with the higher carotid intima-media thickness in type 2 diabetic subjects. Endocrine journal. 2011;58:841-7.

[46] Zhang WX, Zhou W, Zhang ZM, Zhang ZQ, He JF, Shi BY. Decreased retinol-binding protein 4 in the sera of patients with end-stage renal disease after kidney transplantation. Genetics and molecular research : GMR. 2014;13:8126-34.

[47] Pelletier CC, Koppe L, Alix PM, Kalbacher E, Croze ML, Hadj-Aissa A, et al. The relationship between renal function and plasma concentration of the cachectic factor zinc-alpha2-glycoprotein (ZAG) in adult patients with chronic kidney disease. PLoS ONE. 2014;9:e103475.

[48] Sorensen-Zender I, Bhayana S, Susnik N, Rolli V, Batkai S, Baisantry A, et al. Zinc-alpha2-Glycoprotein Exerts Antifibrotic Effects in Kidney and Heart. J Am Soc Nephrol. 2015;26:2659-68.

[49] Feingold KR, Grunfeld C. Effect of inflammation on HDL structure and function. Curr Opin Lipidol. 2016;27:521-30. [50] Zimetti F, De Vuono S, Gomaraschi M, Adorni MP, Favari E, Ronda N, et al. Plasma cholesterol homeostasis, HDL remodeling and function during the acute phase reaction. J Lipid Res. 2017;58:2051-60.

[51] Wait R, Chiesa G, Parolini C, Miller I, Begum S, Brambilla D, et al. Reference maps of mouse serum acute-phase proteins: Changes with LPS-induced inflammation and apolipoprotein A-I and A-II transgenes. Proteomics. 2005;5:4245-53.

[52] Wang W, Xu H, Shi Y, Nandedkar S, Zhang H, Gao H, et al. Genetic deletion of apolipoprotein A-I increases airway hyperresponsiveness, inflammation, and collagen deposition in the lung. J Lipid Res. 2010;51:2560-70.

\section{Addendum to Materials and Methods}

\section{Mass spectrometry identification of proteins from 2-DE gels}

The gel pieces were firstly washed and destained with $25 \mathrm{mmol} / \mathrm{L} \mathrm{NH}_{4} \mathrm{HCO}_{3}$ and $50 \% \mathrm{v} / \mathrm{v}$ acetonitrile

$(\mathrm{ACN})$ twice, then three times with $1: 2 \mathrm{ACN} / 50 \mathrm{mmol} / \mathrm{L} \mathrm{NH}_{4} \mathrm{HCO}_{3}$. Finally the gel pieces were

dehydrated with ACN and dried in SpeedVac for about 20 minutes.

Samples were removed from SpeedVac and trypsin (Promega, Milan, Italy) at $12 \mu \mathrm{g} / \mathrm{ml}$ in $50 \mathrm{mmol} / \mathrm{L}$

$\mathrm{NH}_{4} \mathrm{HCO}_{3}$ was added for 30 minutes in ice. After the trypsin absorption the gel pieces were completely

covered with few microliters of $50 \mathrm{mmol} / \mathrm{L} \mathrm{NH}_{4} \mathrm{HCO}_{3}$ and put overnight at $37^{\circ} \mathrm{C}$. After incubation formic acid was added at a final concentration of $0.1 \%$ and the products were analyzed by mass spectrometry. The samples were analysed by means of LC-ESI-MS/MS, with the spectra being recorded by a hybrid quadrupole orthogonal acceleration time-of-flight (Q-Tof) mass spectrometer (SYNAPT-MS G1, Waters Corporation, Milford, MA, USA) equipped with a TRIZAIC source and connected to a nanoACQUITY 
UPLC system. The samples were injected onto a TRIZAIC nanoTile (Waters Corporation, Milford, MA, USA), Acquity HSS T3, that integrates a trapping column $(5 \mu \mathrm{m}, 180 \mu \mathrm{m} \times 20 \mathrm{~mm})$ for desalting and an analytical column $(1.8 \mu \mathrm{m}, 85 \mu \mathrm{m} \times 100 \mathrm{~mm})$ for peptide separation with an high level of reproducibility of retention time. The elution was performed at a flow rate of $450 \mathrm{nl} / \mathrm{min}$ by increasing the organic solvent concentration from 3 to $40 \%$ B in 30 min, using $0.1 \%$ formic acid in water as reversed phase solvent $\mathrm{A}$ and $0.1 \%$ formic acid in $\mathrm{ACN}$ as reversed phase solvent $\mathrm{B}$. The Tof analyzer was externally calibrated using [Glu1]-fibrinopeptide B from m/z 50 to 1990, and the data were post-acquisition lockmass corrected using the monoisotopic mass of the doubly charged precursor of [ $\left.\mathrm{Glu}^{1}\right]$-Fibrinopeptide B $(\mathrm{m} / \mathrm{z} 785.8426)$ infused into the mass spectrometer at a flow rate of $100 \mathrm{nl} / \mathrm{min}$ through a NanoLockSpray interface using the auxiliary pump of a nanoACQUITY system. A survey scan over the $m / z$ range of 3501990 was used to identify protonated peptides with charge states of 2,3 or 4 , which were automatically selected for data-dependent MS/MS analysis (MassLynx v 4.1 SCN833, Waters Corporation, Milford, MA, USA). All raw MS data were processed with ProteinLynx Global SERVER software (PLGS v 2.5.3, Waters Corporation, Milford, MA, USA) and the proteins were identified by correlating the interpreted spectra with entries in UniProt database.

A UniProt database (release 2015-3; number of human sequence entries, 20199) was used for database searches of each run. Carbamidomethylation was considered as fixed modification and methionine oxidation as variable, one missed cleavage per peptide was allowed, and the mass tolerance window was set to $25 \mathrm{ppm}$ for peptide precursors and 0.05 Da for fragments. In parallel, the spectra were also searched against Uniprot database using Mascot (Matrix Science, London, UK). Valid identification required two or more peptides independently matching the same protein sequence, with a significant peptide score (higher than the identity score from Mascot). 


\section{Supplementary Table 2}

Demographic and lipid/lipoprotein profile of carriers of LCAT deficiency used for serum haptoglobin determination by ELISA.

Carriers of LCAT deficiency

$P$ trend

\begin{tabular}{|c|c|c|c|c|}
\hline & Controls & Heterozygote & Homozygotes & \\
\hline$N$ & 23 & 25 & 13 & \\
\hline $\operatorname{Sex}(m / f)$ & $9 / 14$ & $13 / 12$ & $10 / 3$ & \\
\hline Age (years) & $45.0 \pm 26.1$ & $50.5 \pm 21.0$ & $34.6 \pm 11.2$ & 0.109 \\
\hline Total Cholesterol (mg/dL) & $197.1 \pm 43.3$ & $167.2 \pm 37.2$ & $160.8 \pm 83.5$ & 0.071 \\
\hline Unesterified Cholesterol (mg/dL) & $54.3 \pm 11.7$ & $47.6 \pm 12.5$ & $143.9 \pm 71.1$ & $<0.001$ \\
\hline Unesterified/total Cholesterol & $0.28 \pm 0.04$ & $0.29 \pm 0.06$ & $0.92 \pm 0.11$ & $<0.001$ \\
\hline LDL-Cholesterol (mg/dL) & $117.8 \pm 33.5$ & $101.5 \pm 32.5$ & $95.0 \pm 63.0$ & 0.220 \\
\hline HDL-Cholesterol (mg/dL) & $60.5 \pm 14.3$ & $39.6 \pm 12.3$ & $7.8 \pm 4.4$ & $<0.001$ \\
\hline Triglycerides (mg/dL) & $94.7 \pm 50.6$ & $132.6 \pm 55.3$ & $279.8 \pm 198.6$ & $<0.001$ \\
\hline Apolipoprotein A-I (mg/dL) & $132.0 \pm 27.7$ & $103.6 \pm 23.0$ & $38.5 \pm 9.4$ & $<0.001$ \\
\hline Apolipoprotein A-II (mg/dL) & $31.8 \pm 6.6$ & $30.4 \pm 5.1$ & $6.7 \pm 3.7$ & $<0.001$ \\
\hline Apolipoprotein B (mg/dL) & $92.2 \pm 25.3$ & $93.6 \pm 20.4$ & $52.2 \pm 30.9$ & $<0.001$ \\
\hline preß-HDL (\% of apoA-I) & $12.9 \pm 2.9$ & $18.8 \pm 7.6$ & $45.2 \pm 10.8$ & $<0.001$ \\
\hline LCAT mass $(\mu \mathrm{g} / \mathrm{mL})$ & $5.0 \pm 1.0$ & $3.7 \pm 1.4$ & $1.5 \pm 1.0$ & $<0.001$ \\
\hline LCAT activity (nmol/mL/h) & $41.1 \pm 11.8$ & $20.1 \pm 12.3$ & nd & $<0.001$ \\
\hline
\end{tabular}

Data are reported as mean \pm SD. $n d=$ not detectable 